\title{
SOLUTION OF NONLINEAR FREDHOLM-HAMMERSTEIN INTEGRAL EQUATIONS BY USING SEMIORTHOGONAL SPLINE WAVELETS
}

\author{
M. LAKESTANI, M. RAZZAGHI, AND M. DEHGHAN
}

Received 16 June 2004

Compactly supported linear semiorthogonal B-spline wavelets together with their dual wavelets are developed to approximate the solutions of nonlinear Fredholm-Hammerstein integral equations. Properties of these wavelets are first presented; these properties are then utilized to reduce the computation of integral equations to some algebraic equations. The method is computationally attractive, and applications are demonstrated through an illustrative example.

\section{Introduction}

Wavelets theory is a relatively new and emerging area in mathematical research. It has been applied in a wide range of engineering disciplines; particularly, wavelets are very successfully used in signal analysis for waveform representations and segmentations, timefrequency analysis, and fast algorithms for easy implementation [6]. Wavelets permit the accurate representation of a variety of functions and operators. Moreover, wavelets establish a connection with fast numerical algorithms [2, 3]. Wavelets can be separated into two distinct types, orthogonal and semiorthogonal [5]. Publications on integral equation methods have shown a marked preference for orthogonal wavelets [11]. This is probably because the original wavelets, which were widely used for signal processing, were primarily orthogonal. In signal processing applications, unlike integral equation methods, the wavelet itself is never constructed since only its scaling function and coefficients are needed. However, orthogonal wavelets either have infinite support or a nonsymmetric, and in some cases fractal, nature. These properties can make them a poor choice for characterization of a function. In contrast, the semiorthogonal wavelets have finite support, both even and odd symmetry, and simple analytical expressions, ideal attributes of a basis function [11].

Several numerical methods for approximating the solution of Hammerstein integral equations are known. For Fredholm-Hammerstein integral equations, the classical method of successive approximations was introduced in [12]. A variation of the Nyström method was presented in [10]. A collocation-type method was developed in [9]. In [4], Brunner applied a collocation-type method to nonlinear Volterra-Hammerstein integral 
equations and integrodifferential equations, and discussed its connection with the iterated collocation method. Guoqiang [8] introduced and discussed the asymptotic error expansion of a collocation-type method for Volterra-Hammerstein integral equations. The methods in $[8,9]$ transform a given integral equation into a system of nonlinear equations, which has to be solved with some kind of an iterative method. In [9] the definite integrals involved in the solution may be evaluated analytically only in favorable cases, while in [8] the integrals involved in the solution have to be evaluated at each time step of the iteration.

In the present paper, we apply compactly supported linear semiorthogonal B-spline wavelets, specially constructed for the bounded interval to solve the nonlinear FredholmHammerstein integral equations of the form

$$
y(x)=f(x)+\int_{0}^{1} K(x, t) g[t, y(t)] d t, \quad 0 \leq x \leq 1,
$$

where $f, g$, and $K$ are given continuous functions, with $g(t, y)$ nonlinear in $y$. The use of semiorthogonal compactly supported spline wavelets is justified by their interesting properties. Among them, the following can be explicitly cited [1]: they satisfy all the properties on a bounded interval that are verified by the usual wavelets on the real line, but they do not present the difficulties related to the boundary conditions, when applying such wavelets to problems in finite bounded domains, unlike most of the continuous orthogonal wavelets. Also, the semiorthogonal compactly supported spline wavelets have closedform expressions. In [11], the two categories of wavelets, orthogonal and semiorthogonal, are compared, and it is shown that semiorthogonal wavelets are best suited for integral equation applications.

Our method consists of reducing (1.1) to a set of algebraic equations by expanding the unknown function as linear B-spline wavelets with unknown coefficients. The properties of these wavelets are then utilized to evaluate the unknown coefficients. The paper is organized as follows. In Section 2, we describe the formulation of the B-spline scaling functions and wavelets on $[0,1]$ required for our subsequent development. In Section 3, the proposed method is used to approximate the solution of nonlinear FredholmHammerstein integral equation. In Section 4, we report our numerical finding and demonstrate the accuracy of the proposed numerical scheme by considering a numerical example.

\section{B-spline scaling functions and wavelets on $[0,1]$}

When semiorthogonal wavelets are constructed from B-splines of order $m$, the lowest octave level $j=j_{0}$ is determined in [7] by

$$
2^{j_{0}} \geq 2 m-1
$$

so as to give a minimum of one complete wavelet on the interval $[0,1]$. In this paper, we will use a wavelet generated by a linear spline-the second-order cardinal B-spline basis function. From (2.1), the second-order B-spline of lowest level, which must be an integer, is determined to be $j_{0}=2$. This constrains all octave levels to $j \geq 2$. 
As is the case with all semiorthogonal wavelets, the second-order B-splines also serve as scaling functions. The second-order B-splines/scaling functions are given by

$$
\phi_{j, k}(x)= \begin{cases}x_{j}-k, & k \leq x_{j} \leq k+1, \\ 2-\left(x_{j}-k\right), & k+1 \leq x_{j} \leq k+2, k=0, \ldots, 2^{j}-2, \\ 0, & \text { otherwise }\end{cases}
$$

with the respective left- and right-hand side boundary scaling functions

$$
\begin{aligned}
& \phi_{j, k}(x)= \begin{cases}2-\left(x_{j}-k\right), & 0 \leq x_{j} \leq 1, k=-1, \\
0, & \text { otherwise, }\end{cases} \\
& \phi_{j, k}(x)= \begin{cases}x_{j}-k, & k \leq x_{j} \leq k+1, k=2^{j}-1, \\
0, & \text { otherwise. }\end{cases}
\end{aligned}
$$

The actual coordinate position $x$ is related to $x_{j}$ according to $x_{j}=2^{j} x$. The second-order B-spline wavelets are given by

$$
\psi_{j, k}(x)=\frac{1}{6} \begin{cases}x_{j}-k, & k \leq x_{j} \leq k+\frac{1}{2}, \\ 4-7\left(x_{j}-k\right), & k+\frac{1}{2} \leq x_{j} \leq k+1, \\ -19+16\left(x_{j}-k\right), & k+1 \leq x_{j} \leq k+\frac{3}{2} \\ 29-16\left(x_{j}-k\right), & k+\frac{3}{2} \leq x_{j} \leq k+2, k=0, \ldots, 2^{j}-3, \\ -17+7\left(x_{j}-k\right), & k+2 \leq x_{j} \leq k+\frac{5}{2} \\ 3-\left(x_{j}-k\right), & k+\frac{5}{2} \leq x_{j} \leq k+3 \\ 0, & \text { otherwise }\end{cases}
$$

with the respective left- and right-hand side boundary wavelets

$$
\psi_{j, k}(x)=\frac{1}{6} \begin{cases}-6+23 x_{j}, & 0 \leq x_{j} \leq \frac{1}{2}, \\ 14-17 x_{j}, & \frac{1}{2} \leq x_{j} \leq 1, \\ -10+7 x_{j}, & 1 \leq x_{j} \leq \frac{3}{2}, k=-1, \\ 2-x_{j}, & \frac{3}{2} \leq x_{j} \leq 2, \\ 0, & \text { otherwise, }\end{cases}
$$


116 Fredholm-Hammerstein equations by using wavelets

$$
\psi_{j, k}(x)=\frac{1}{6} \begin{cases}2-\left(k+2-x_{j}\right), & k \leq x_{j} \leq k+\frac{1}{2}, \\ -10+7\left(k+2-x_{j}\right), & k+\frac{1}{2} \leq x_{j} \leq k+1, \\ 14-17\left(k+2-x_{j}\right), & k+1 \leq x_{j} \leq k+\frac{3}{2}, k=2^{j}-2, \\ -6+23\left(k+2-x_{j}\right), & k+\frac{3}{2} \leq x_{j} \leq k+2, \\ 0, & \text { otherwise. }\end{cases}
$$

For example, for $j=2$, the inner scaling functions are obtained by putting $k=0,1,2$ in (2.2) as

$$
\begin{aligned}
& \phi_{2,0}(x)= \begin{cases}4 x, & 0 \leq x<\frac{1}{4}, \\
2-4 x, & \frac{1}{4} \leq x<\frac{1}{2}, \\
0, & \text { otherwise, }\end{cases} \\
& \phi_{2,1}(x)= \begin{cases}4 x-1, & \frac{1}{4} \leq x \leq \frac{1}{2}, \\
1-4 x, & \frac{1}{2} \leq x \leq \frac{3}{4}, \\
0, & \text { otherwise, }\end{cases} \\
& \phi_{2,2}(x)= \begin{cases}4 x-2, & \frac{1}{2} \leq x \leq \frac{3}{4}, \\
-4 x, & \frac{3}{4} \leq x \leq 1, \\
0, & \text { otherwise. }\end{cases}
\end{aligned}
$$

Also, for $j=2$, the left- and right-hand side boundary scaling functions are obtained by putting $j=2, k=-1$, and $k=3$ in (2.3) and (2.4), respectively, as

$$
\begin{gathered}
\phi_{2,-1}(x)= \begin{cases}1-4 x, & 0 \leq x \leq \frac{1}{4} \\
0, & \text { otherwise }\end{cases} \\
\phi_{2,3}(x)= \begin{cases}4 x-3, & \frac{3}{4} \leq x \leq 1 \\
0, & \text { otherwise }\end{cases}
\end{gathered}
$$

Similarly, for $j=2$, the inner wavelet functions are obtained by putting $j=2, k=0$, and $k=1$ in (2.5) and the left- and right-hand side boundary wavelets are obtained by putting $j=2, k=-1$, and $k=2$ in (2.6) and (2.7), respectively. 
2.1. Function approximation. A function $f(x)$ defined over $[0,1]$ may be represented by B-spline wavelets as

$$
f(x)=\sum_{k=-1}^{3} c_{k} \phi_{2, k}+\sum_{i=2}^{\infty} \sum_{j=-1}^{2^{(i-1)}} d_{i, j} \psi_{i, j}
$$

where $\phi_{2, k}$ and $\psi_{i, j}$ are scaling and wavelets functions, respectively. If the infinite series in (2.13) is truncated, then (2.13) can be written as

$$
f(x)=\sum_{k=-1}^{3} c_{k} \phi_{2, k}+\sum_{i=2}^{M} \sum_{j=-1}^{2^{(i-1)}} d_{i, j} \psi_{i, j}=C^{T} \Psi,
$$

where $C$ and $\Psi$ are $\left(2^{(M+1)}+1\right) \times 1$ vectors given by

$$
\begin{aligned}
C & =\left[c_{-1}, c_{0}, \ldots, c_{3}, d_{2,-1}, \ldots, d_{2,2}, d_{3,-1}, \ldots, d_{3,6}, \ldots, d_{M,-1}, \ldots, d_{M, 2^{(M-1)}}\right]^{T}, \\
\Psi & =\left[\phi_{2,-1}, \phi_{2,0}, \ldots, \phi_{2,3}, \psi_{2,-1}, \ldots, \psi_{2,2}, \psi_{3,-1}, \ldots, \psi_{3,6}, \ldots, \psi_{M,-1}, \ldots, \psi_{M, 2^{(M-1)}}\right]^{T},
\end{aligned}
$$

with

$$
\begin{aligned}
c_{k} & =\int_{0}^{1} f(x) \tilde{\phi}_{2, k}(x) d x, \quad k=-1,0, \ldots, 3 \\
d_{i, j} & =\int_{0}^{1} f(x) \tilde{\psi}_{i, j}(x) d x, \quad i=2,3,4, \ldots, M, j=-1,0,1, \ldots, 2^{(i-1)},
\end{aligned}
$$

where $\tilde{\phi}_{2, k}(x)$ and $\tilde{\psi}_{i, j}(x)$ are dual functions of $\phi_{2, k}$, and $\psi_{i, j}$, respectively. These can be obtained by linear combinations of $\phi_{2, k}, k=-1, \ldots, 3$, and $\psi_{i, j}, i=2, \ldots, M, j=-1, \ldots$, $2^{(M-1)}$, as follows. Let

$$
\begin{aligned}
& \Phi=\left[\phi_{2,-1}(x), \phi_{2,0}(x), \phi_{2,1}(x), \phi_{2,2}(x), \phi_{2,3}(x)\right]^{T}, \\
& \bar{\Psi}=\left[\psi_{2,-1}(x), \psi_{2,0}(x), \ldots, \psi_{M, 2^{(M-1)}}(x)\right]^{T} .
\end{aligned}
$$

Using (2.8)-(2.12) and (2.19) we get

$$
\int_{0}^{1} \Phi \Phi^{T} d x=P_{1}=\left[\begin{array}{ccccc}
\frac{1}{12} & \frac{1}{24} & 0 & 0 & 0 \\
\frac{1}{24} & \frac{1}{6} & \frac{1}{24} & 0 & 0 \\
0 & \frac{1}{24} & \frac{1}{6} & \frac{1}{24} & 0 \\
0 & 0 & \frac{1}{24} & \frac{1}{6} & \frac{1}{24} \\
0 & 0 & 0 & \frac{1}{24} & \frac{1}{12}
\end{array}\right]
$$


118 Fredholm-Hammerstein equations by using wavelets

and from (2.5)-(2.7) and (2.20) we have

$$
\int_{0}^{1} \bar{\Psi} \bar{\Psi}^{T} d x=P_{2}=\left[\begin{array}{cccc}
N_{4 \times 4} & & & \\
& \frac{1}{2} N_{8 \times 8} & & \\
& & \ddots & \\
& & & \frac{1}{2^{M-2}} N_{2^{M} \times 2^{M}}
\end{array}\right],
$$

where $P_{1}$ and $P_{2}$ are $5 \times 5$ and $\left(2^{M+1}-4\right) \times\left(2^{M+1}-4\right)$ matrices, respectively, and $N$ is a five-diagonal matrix given by

$$
N=\left[\begin{array}{ccccccc}
\frac{2}{27} & \frac{1}{96} & -\frac{-1}{864} & 0 & 0 & \ldots & 0 \\
\frac{1}{96} & \frac{1}{16} & \frac{5}{432} & -\frac{1}{864} & 0 & \ldots & 0 \\
-\frac{1}{864} & \frac{5}{432} & \frac{1}{16} & \frac{5}{432} & -\frac{1}{864} & \cdots & 0 \\
\vdots & \ddots & \ddots & \ddots & \ddots & \ddots & \vdots \\
0 & \cdots & -\frac{1}{864} & \frac{5}{432} & \frac{1}{16} & \frac{5}{432} & -\frac{1}{864} \\
0 & \cdots & 0 & -\frac{1}{864} & \frac{5}{432} & \frac{1}{16} & \frac{1}{96} \\
0 & \cdots & 0 & 0 & -\frac{1}{864} & \frac{1}{96} & \frac{2}{27}
\end{array}\right] .
$$

Suppose $\tilde{\Phi}$ and $\tilde{\Psi}$ are the dual functions of $\Phi$ and $\bar{\Psi}$, respectively, given by

$$
\begin{aligned}
& \tilde{\Phi}=\left[\tilde{\phi}_{2,-1}(x), \tilde{\phi}_{2,0}(x), \tilde{\phi}_{2,1}(x), \tilde{\phi}_{2,2}(x), \tilde{\phi}_{2,3}(x)\right]^{T}, \\
& \tilde{\Psi}=\left[\tilde{\psi}_{2,-1}(x), \tilde{\psi}_{2,0}(x), \ldots, \tilde{\psi}_{M, 2^{M}-2}(x)\right]^{T} .
\end{aligned}
$$

Using (2.17)-(2.20), (2.24), and (2.25) we have

$$
\int_{0}^{1} \tilde{\Phi} \Phi^{T} d x=I_{1}, \quad \int_{0}^{1} \tilde{\tilde{\Psi}} \bar{\Psi}^{T} d x=I_{2},
$$

where $I_{1}$ and $I_{2}$ are $5 \times 5$ and $\left(2^{(M+1)}-4\right) \times\left(2^{(M+1)}-4\right)$ identity matrices, respectively. Then (2.21), (2.22), and (2.26) give

$$
\tilde{\Phi}=P_{1}^{-1} \Phi, \quad \tilde{\bar{\Psi}}=P_{2}^{-1} \bar{\Psi} .
$$




\section{Nonlinear Fredholm-Hammerstein integral equations}

In this section, we solve nonlinear Fredholm-Hammerstein integral equations of the form in (1.1) by using B-spline wavelets. For this purpose, we first assume

$$
z(x)=g(x, y(x)), \quad 0 \leq x \leq 1 .
$$

We now use (2.14) to approximate $y(x), z(x)$ as

$$
y(x)=D^{T} \Psi(x), \quad z(x)=E^{T} \Psi(x),
$$

where $\Psi(x)$ is defined in $(2.15)$ and $D$ and $E$ are $\left(2^{(M+1)}+1\right) \times 1$ unknown vectors defined similarly to $C$ in (2.16). We also expand $f(x), K(x, t)$ by B-spline dual wavelets $\tilde{\Psi}$ defined as in (2.24) and (2.25) as

$$
f(x)=\Lambda^{T} \tilde{\Psi}(x), \quad K(x, t)=\tilde{\Psi}^{T}(t) \Theta \tilde{\Psi}(x),
$$

where

$$
\Theta_{(i, j)}=\int_{0}^{1}\left[\int_{0}^{1} K(x, t) \Psi_{i}(t) d t\right] \Psi_{j}(x) d x .
$$

From (3.1), (3.2), and (3.3) we get

$$
\begin{aligned}
\int_{0}^{1} K(x, t) g(t, y(t)) d t & =\int_{0}^{1} E^{T} \Psi(t) \tilde{\Psi}^{T}(t) \Theta \tilde{\Psi}(x) d t \\
& =E^{T}\left[\int_{0}^{1} \Psi(t) \tilde{\Psi}^{T}(t) d t\right] \Theta \tilde{\Psi}(x) \\
& =E^{T} \Theta \tilde{\Psi}(x) .
\end{aligned}
$$

Applying (3.1)-(3.5) in (1.1), we get

$$
D^{T} \Psi(x)-\Lambda^{T} \tilde{\Psi}(x)-E^{T} \Theta \tilde{\Psi}(x)=0 ;
$$

multiplying (3.6) by $\Psi^{T}(x)$ and integrating from 0 to 1 , we have

$$
D^{T} P-\Lambda^{T}-E^{T} \Theta=0,
$$

in which $P$ is a $\left(2^{(M+1)}+1\right) \times\left(2^{(M+1)}+1\right)$ square matrix given by

$$
P=\int_{0}^{1} \Psi(x) \Psi^{T}(x) d x=\left[\begin{array}{ll}
P_{1} & \\
& P_{2}
\end{array}\right] .
$$

To find the solution $y(x)$ in (3.2), we first collocate the following equation in $x_{i}=i / 2^{M+1}$, $i=0,1, \ldots, 2^{M+1}$ :

$$
g\left(x, D^{T} \Psi(x)\right)=E^{T} \Psi(x)
$$

Equation (3.7) generates a set of $2^{(M+1)}+1$ algebraic equations. The total number of unknowns for vectors $D$ and $E$ in $(3.2)$ is $2\left[2^{(M+1)}+1\right]$. These can be obtained by using (3.7) and (3.9). 
120 Fredholm-Hammerstein equations by using wavelets

Table 4.1. Exact and approximate solutions.

\begin{tabular}{ccccc}
\hline x & $\begin{array}{c}\text { Approximate } \\
M=2\end{array}$ & $\begin{array}{c}\text { Approximate } \\
M=4\end{array}$ & $\begin{array}{c}\text { Approximate } \\
M=6\end{array}$ & Exact \\
\hline 0 & 1 & 1 & 1 & 1 \\
0.1 & 0.995688 & 0.995012 & 0.995005 & 0.995004 \\
0.2 & 0.983165 & 0.983077 & 0.983094 & 0.983095 \\
0.3 & 0.955652 & 0.955324 & 0.955336 & 0.955336 \\
0.4 & 0.921345 & 0.921066 & 0.921062 & 0.921061 \\
0.5 & 0.877261 & 0.877575 & 0.877582 & 0.877583 \\
0.6 & 0.825418 & 0.825343 & 0.825337 & 0.825336 \\
0.7 & 0.764678 & 0.764859 & 0.764844 & 0.764842 \\
0.8 & 0.696157 & 0.696694 & 0.696705 & 0.696707 \\
0.9 & 0.621445 & 0.621603 & 0.621617 & 0.621619 \\
1 & 0.540280 & 0.540312 & 0.540303 & 0.540302 \\
\hline
\end{tabular}

\section{Illustrative example}

Consider the equation

$$
y(x)=1+3 \sin ^{2}(x)+\int_{0}^{1} K(x, t) y^{2}(t) d t, \quad 0 \leq x \leq 1,
$$

where

$$
K(x, t)= \begin{cases}-3 \sin (x-t), & 0 \leq t \leq x, \\ 0, & x<t \leq 1 .\end{cases}
$$

The solution for $y(x)$ is obtained by the method in Section 3. The computational results for $M=2, M=4$, and $M=6$ together with the exact solution $y(x)=\cos (x)$ are given in Table 4.1.

\section{Conclusion}

In the present work, a technique has been developed for solving nonlinear FredholmHammerstein integral equations. The method is based upon compactly supported linear semiorthogonal B-spline wavelets. The dual wavelets for these B-spline wavelets were also given. The problem has been reduced to solving a system of nonlinear algebraic equations. An illustrative example was included to demonstrate the validity and applicability of the technique. 


\section{References}

[1] G. Ala, M. L. D Silvestre, E. Francomano, and A. Tortorici, An advanced numerical model in solving thin-wire integral equations by using semi-orthogonal compactly supported spline wavelets, IEEE Trans. Electromagn. Compat. 45 (2003), no. 2, 218-228.

[2] B. K. Alpert, Wavelets and other bases for fast numerical linear algebra, Wavelets: A Tutorial Theory and Applications (C. K. Chui, ed.), Wavelet Anal. Appl., vol. 2, Academic Press, Massachusetts, 1992, pp. 181-216.

[3] G. Beylkin, R. Coifman, and V. Rokhlin, Fast wavelet transforms and numerical algorithms. I, Commun. Pure Appl. Math. 44 (1991), no. 2, 141-183.

[4] H. Brunner, Implicitly linear collocation methods for nonlinear Volterra equations, Appl. Numer. Math. 9 (1992), no. 3-5, 235-247.

[5] C. K. Chui, An Introduction to Wavelets, Wavelet Analysis and Its Applications, vol. 1, Academic Press, Massachusetts, 1992.

[6] Wavelets: A Mathematical Tool for Signal Analysis, SIAM Monographs on Mathematical Modeling and Computation, Society for Industrial and Applied Mathematics, Pennsylvania, 1997.

[7] J. C. Goswami, A. K. Chan, and C. K. Chui, On solving first-kind integral equations using wavelets on a bounded interval, IEEE Trans. Antennas Propagat. 43 (1995), no. 6, 614-622.

[8] H. Guoqiang, Asymptotic error expansion of a collocation-type method for Volterra-Hammerstein integral equations, Appl. Numer. Math. 13 (1993), no. 5, 357-369.

[9] S. Kumar and I. H. Sloan, A new collocation-type method for Hammerstein integral equations, Math. Comp. 48 (1987), no. 178, 585-593.

[10] L. J. Lardy, A variation of Nyström's method for Hammerstein equations, J. Integral Equations 3 (1981), no. 1, 43-60.

[11] R. D. Nevels, J. C. Goswami, and H. Tehrani, Semi-orthogonal versus orthogonal wavelet basis sets for solving integral equations, IEEE Trans. Antennas Propagat. 45 (1997), no. 9, 1332-1339.

[12] F. G. Tricomi, Integral Equations, Dover, New York, 1982.

M. Lakestani: Department of Applied Mathematics, Amirkabir University of Technology, Tehran 15914, Iran

E-mail address: lakestani@aut.ac.ir

M. Razzaghi: Department of Mathematics and Statistics, Mississippi State University, MS 39762, USA; Department of Applied Mathematics, Amirkabir University of Technology, Tehran 15914, Iran

E-mail address: razzaghi@math.msstate.edu

M. Dehghan: Department of Applied Mathematics, Amirkabir University of Technology, Tehran 15914, Iran

E-mail address: mdehghan@cic.aut.ac.ir 


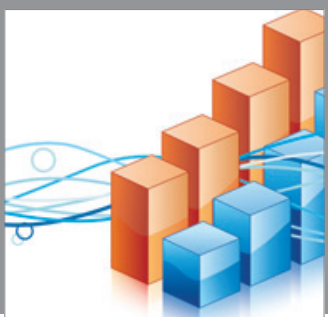

Advances in

Operations Research

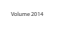

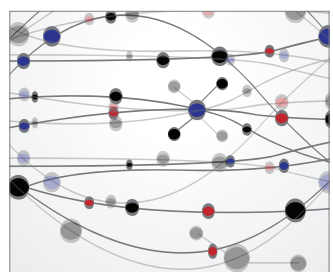

\section{The Scientific} World Journal
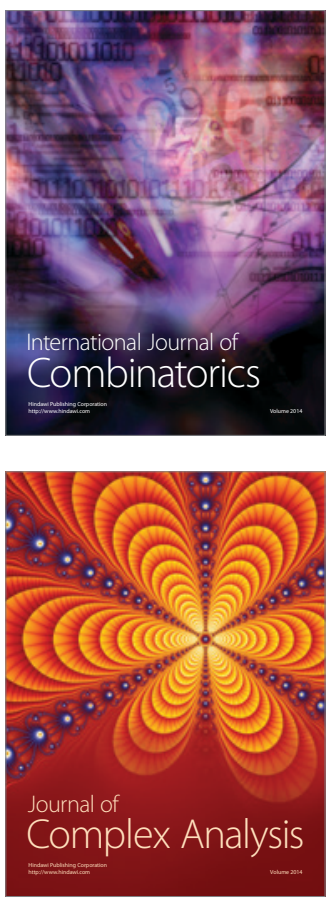

International Journal of

Mathematics and

Mathematical

Sciences
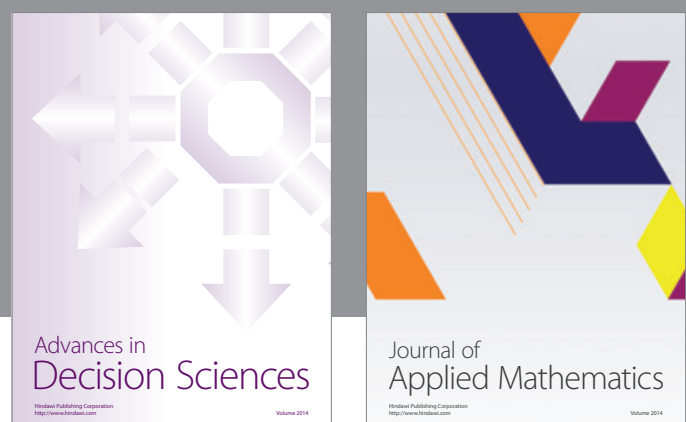

Journal of

Applied Mathematics
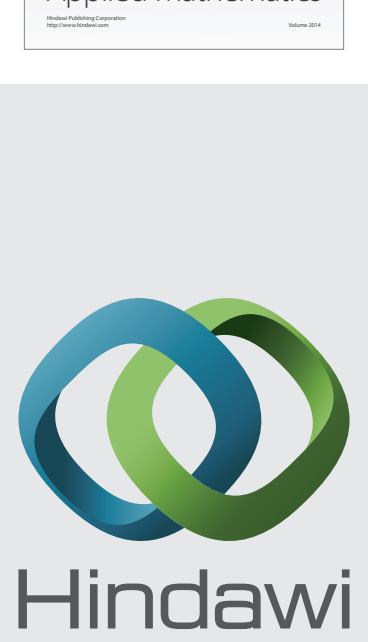

Submit your manuscripts at http://www.hindawi.com
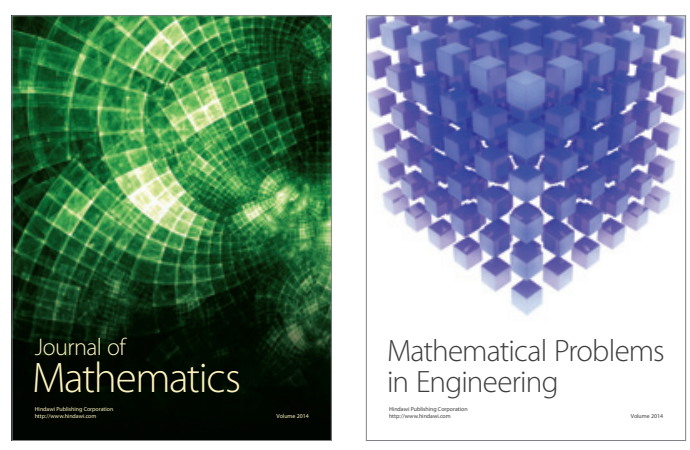

Mathematical Problems in Engineering
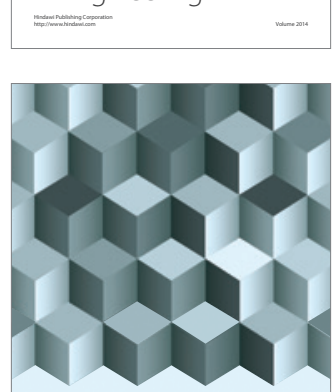

Journal of

Function Spaces
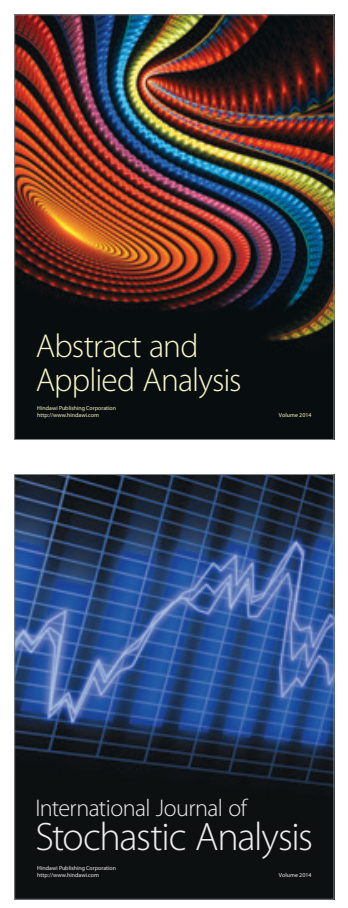

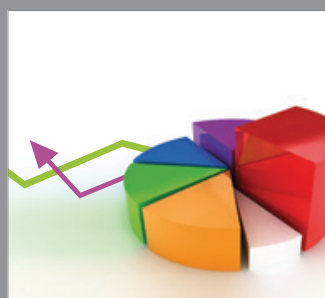

ournal of

Probability and Statistics

Promensencen
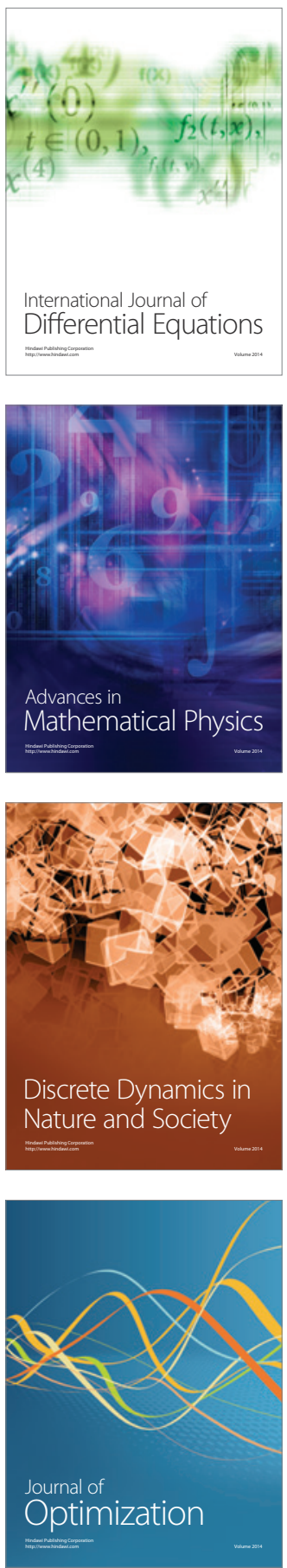